\title{
Studying Host-microbiota Mutualism in Drosophila: Harnessing the Power of Gnotobiotic Flies
}

\author{
Dali Ma, Gilles Storelli, Mélanie Mitchell, François Leulier
}

\begin{abstract}
The complex interaction between the metazoan host and its commensal gut microbiota is one of the essential features of symbiosis in the animal kingdom. As there is a burgeoning interest to decipher the molecular dialog that shapes host-microbiota mutualism, the use of gnotobiotic model organism becomes an imperative approach to unambiguously parse the specific contributions to such interaction from the microbiome. In this
\end{abstract} review, we focus on several remarkable

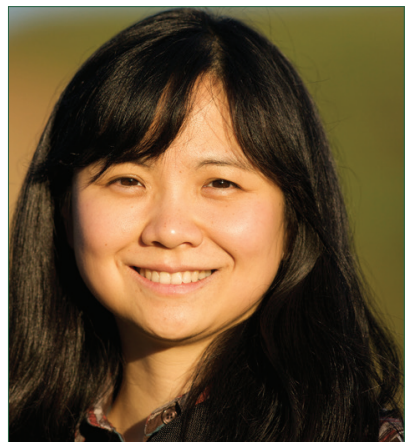

Dr. Dali Ma

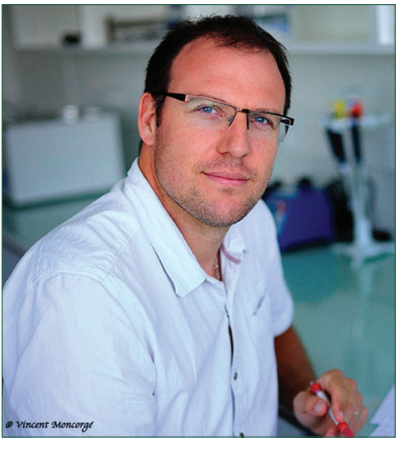

Dr. François Leulier gnotobiotic studies in Drosophila that functionally depicted how the gut microbes can alter host physiology and behavior through transcriptomic regulation, hormonal control, and diet modification. These results in concert illustrate that the gnotobiotic flies mono- or poly-associated with members of its gut microbiota deliver a versatile and powerful model that is amenable to different types of studies ranging from classic genetics to large-scale systems approaches. (Biomed J 2015;38:285-293)

\section{Key words: Drosophila, gnotobiology, microbiota, mutualism}

$I^{\mathrm{n}}$ n 1883, Louis Pasteur expressed his wish to raise a "microbially deprived" young animal on "pure" food from birth, and postulated that "without any preconceived notion.... life under such condition... shall become impossible." ${ }^{[1]}$ Nearly 30 years later, Eugene Wollman at the Pasteur institute in Paris successfully cultured the first germ-free common blow flies (Calliphora vomitoria) and observed that except for certain minor growth delay, the adult flies appeared perfectly normal. ${ }^{[2]}$ At first, Wollman's experiment seemed to have put an end to Pasteur's claim; yet in truth, it was only the beginning. Throughout his productive career as a microbiologist, Wollman probably did not realize that his germ-free blow flies spawned an entire field of animal physiology based on host-microbe interactions; and only when a germ-free life was made possible, the concept of "gnotobiology" could spring to life. In the past century, Pasteur's musing on what life would be like without its resident microbes gradually transformed to a quest to understand how the eukaryotic hosts and their bacterial partners orchestrate the symphony of life, and how such interactions probably profoundly altered the course of our evolutionary history. ${ }^{[3]}$

Microbes occupy every possible ecological niche on earth. A set of particular niches comprise the various internal epithelia of the metazoan hosts, who, through eons of evolution, have forged complex and intricate relationships with this rich and diverse microbial community, called the "microbiota." ${ }^{[3,4]} \mathrm{A}$ human host carries on his body far more microorganisms than his own cells, and these invisible dwellers constitute $1-3 \%$ of his body mass. ${ }^{[5,6]}$ The human gut alone harbors approximately $500-1,000$ bacterial species, ${ }^{[7]}$ and represents the largest mucosal surface where the exchanges between the host and the microbiota take place. In the last decades, many studies together generated a systematic understanding of how the gut microbiota and

From the Institut de Génomique Fonctionnelle de Lyon (IGFL), Ecole Normale Supérieure de Lyon, CNRS UMR 5242, Université Claude Bernard Lyon 1, Lyon, France

Received: Jan. 13, 2015; Accepted: May 26, 2015

Correspondence to: Dr. Dali Ma, Institut de Génomique Fonctionnelle de Lyon (IGFL), Ecole Normale Supérieure de Lyon, CNRS UMR 5242, Université Claude Bernard Lyon 1, France. 46 Allée d'Italie, 69364 Lyon Cedex 07, France. E-mail: dali.ma@ens-lyon.fr Correspondence to: Dr. François Leulier, Institut de Génomique Fonctionnelle de Lyon (IGFL), Ecole Normale Supérieure de Lyon, CNRS UMR 5242, Université Claude Bernard Lyon 1, France. 46 Allée d’Italie, 69364 Lyon Cedex 07, France. E-mail: francois.leulier@ens-lyon.fr

DOI: $10.4103 / 2319-4170.158620$ 
its diverse gene repertoire, called the "microbiome", can configure the fitness parameters of the host; a healthy microbiota can expand the host's metabolic potential, fortify its immune system, promote healthy aging, and even dictate its emotional and psychological well-being. ${ }^{[6,8-11]}$ However, as the community structure and activities of the gut microbiota are extremely sensitive to fluctuations in the environment, perturbations to the microbiota pose significant risks to the host. ${ }^{[12,13]}$ Subtle changes in host immunity, diet, or xenobiotic concentration can disrupt the balance in the gut microbial community, which consequently compromises host fitness. In mammals, microbiome imbalance, or dysbiosis, positively correlates with the onset of obesity, diabetes, colon cancer, ${ }^{[14-16]}$ and human psychiatric disorders such as schizophrenia and autism. ${ }^{[17]}$

Currently, a large amount of research on host-microbiota mutualism employs vertebrate models, yet the high complexity of the microbial composition in the mammalian gut, the difficulty to culture most of these microbial species, and the cost of raising these animals in a strictly sterile environment pose a considerable obstacle. Therefore, to delve deeper into the molecular interplay between the host genome and the microbiome and the environmental contributions to such interplay, a more genetically tractable model organism with simpler and even defined microbiota is an attractive option. Drosophila melanogaster fits these criteria. First of all, the intestinal tract of the fruit fly is anatomically and physiologically similar to the mammalian gut ${ }^{[18]}$ yet the microbial composition is rather simple: Throughout the larval and adult life, the fly gut hosts five to twenty aero-tolerant commensal species, all of which are readily cultured in the laboratory. ${ }^{[19,20]}$ Two families of bacteria: Acetobacteraceae and Lactobacillaceae, dominate the community. ${ }^{[21-26]}$ However, the fly gut microbiota is transient in nature and requires constant replenishment; thus, the community structure and bacterial load fluctuate highly as the flies develop and age. ${ }^{[27-29]}$ Such inconstancy makes it difficult to clearly pinpoint the bacterial genetic factors contributing to host physiology. Therefore, the use of gnotobiotic fly models, in combination with classic genetic approaches and next-generation sequencing, proves to be the new and effective means to study intestinal mutualism with added advantage, because it enables the investigators to inoculate the germ-free subjects with various bacterial strains of predefined quantity and composition. In this setting, the researchers not only can rigorously monitor the phenotypic changes in different aspects of host physiology, but also can robustly correlate and even attribute particular changes in the host to the specific functions from the microbiome, as the genomes of many gut microbiota species are being rapidly sequenced and annotated.$^{[5]}$ Moreover, except for Acetobacter, which are mostly found in insects, ${ }^{[30,31]}$ Lactobacillus species are commensal to mammals. ${ }^{[32-34]}$ Therefore, the results from such gnotobiotic fly studies can be readily translated to mammalian studies. Drosophila models were first used to dissect the genetic networks governing host-pathogen interaction (see review by El Chamy et al. in the same issue). With the same approach, pioneering studies have shown promising results to identify and functionally characterize the genetic components of the molecular crosstalk between Drosophila and its commensal bacteria. In this review, we discuss the findings from the studies using gnotobiotic fly models to unravel the impact of the members of gut microbiota on host metabolism, physiology and behavior [Figure 1].

\section{The making of the gnotobiotic flies}

As mentioned before, in the early 1910s, Eugene Wollman and his colleagues at the Pasteur institute were among the first to raise germ-free animals such as common blow flies, tadpoles, and guinea pigs. Wollman made the first germ-free common blow flies by treating the egg surface with diluted hydrogen peroxide and raising the larvae on sterilized meat substrate. ${ }^{[2]}$ Interestingly, Wollman observed that the germ-free larvae reached normal body size, but at a slower rate. Moreover, these flies seemed slower in movement and less interested in foraging. Therefore, even though the "microbially deprived" life was indeed possible in a sterile environment, the difference between such a life and its conventionally reared (CR) siblings was already observable to the naked eye. In the next few decades, Drosophila melanogaster was attaining a more and more prominent status as a model genetic organism. As a result, in the 1950s and 1960 s, different methods were developed to sterilize Drosophila eggs on a large-scale and keeping axenic fly stocks turned into a routine laboratory practice.

In 1969, Marion Bakula developed the first monoxenic Drosophila model by associating bleached fly eggs with either "native" or "foreign" bacterial strains (Escherichia coli). ${ }^{[35]} \mathrm{In}$ her study, only the "native" bacteria isolated from the fly gut persisted throughout larval development in the fly host, who pupariated at a slightly faster pace than the axenic controls. This is also the first gnotobiotic model to demonstrate that the essential mode of microbial transmission in fruit flies is through larval ingestion of the contaminated chorion. Therefore, thorough dechorionation of the eggs can effectively render a fly stock germ-free. In the next several decades, after trying different sterilizing agents such as antiformin and formalin, ${ }^{[36]}$ researchers found that treatment with common household bleach (diluted sodium hypochloride solution) in combination with ethanol wash is the safe, simple, rapid and effective way to dechorionate the embryo and rid the surface of bacterial "contaminants". However, bleaching alone cannot eliminate intracellular endosymbionts such as Wolbachia, the most widespread insect symbiont whose relationship with the host ranges from parasitism to mutualism. Depending 


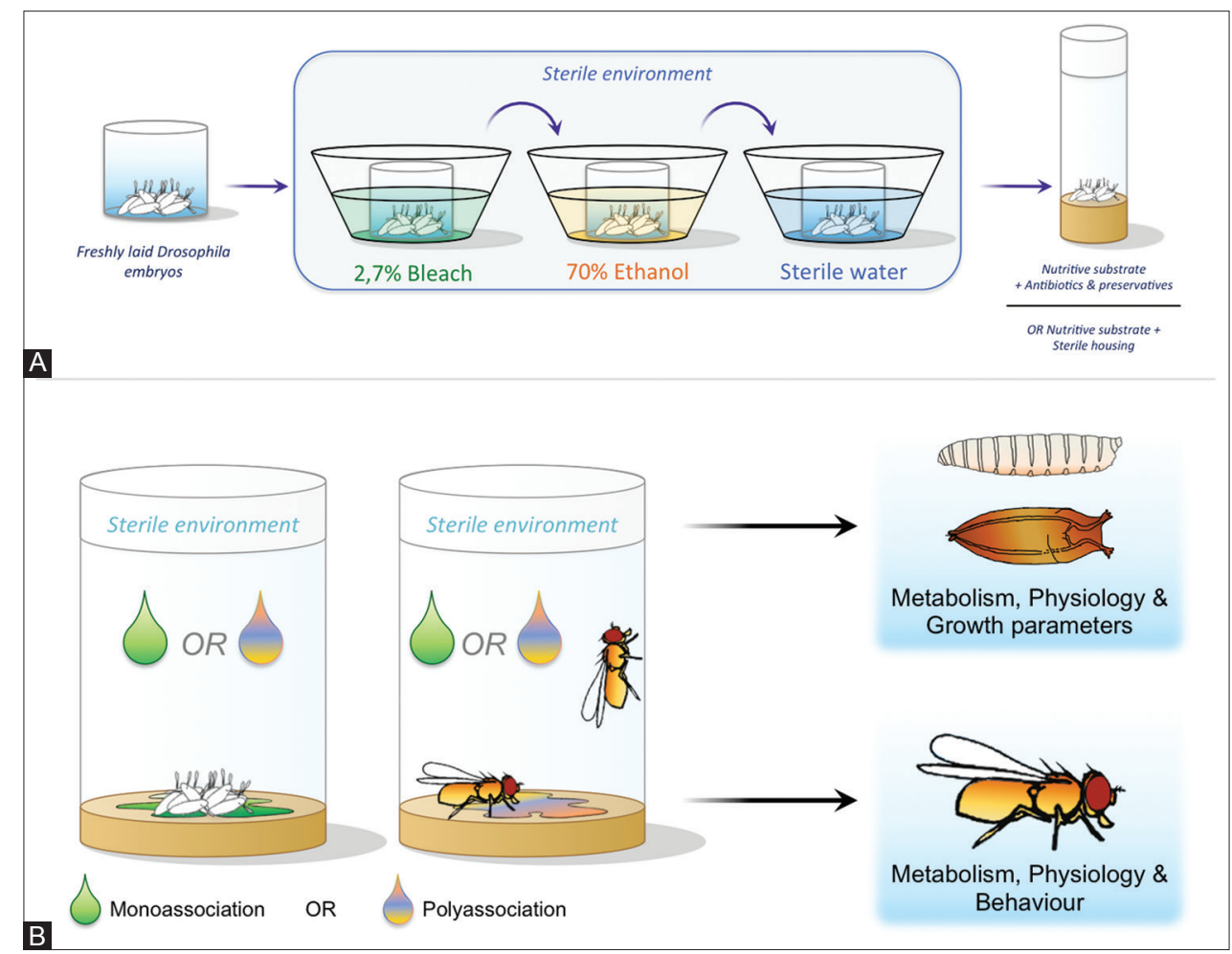

Figure 1: Building a gnotobiotic Drosophila model to study host-microbiota mutualism. (A) To obtain germ-free flies, freshly laid eggs are harvested in a large scale and washed in succession with bleach, ethanol, and sterile water. To maintain axeny, the dechorionated eggs are then grown in the presence of antibiotics and preservatives or in a sterile environment. (B) To study the specific contribution of the microbiome to the different aspects of host physiology, ex-axenic eggs or adults are mono-associated with a single gut commensal species (green drop) or poly-associated with a defined set of gut commensal bacteria (blue and yellow colored drop). Such gnotobiotic flies have been used to study the impact of specific commensals on host juvenile growth, developmental timing, metabolic homeostasis, and adult behavior.

on the context, the presence of Wolbachia is known to affect reproductive success, enhance insulin signaling and boost host defense. ${ }^{[37-40]}$ Therefore, to obtain a "true" germ-free or gut-commensal specific phenotype unadulterated by Wolbachia, different laboratories have adopted various protocols to maintain germ-free stocks, either by combining bleaching with rearing flies on food containing a mixture of antibiotics or by one-time treatment of bleach and the subsequent maintenance of the flies in a sterile environment [Figure 1a]. Of note, bleaching and/or antibiotic treatment can lower fly viability and fecundity and have certain unintended negative cellular and systemic effects on the host. ${ }^{[41]}$ Therefore, the studies using germ-free flies mandate careful and thorough controls. In the following sections, we review a few seminal gnotobiotic Drosophila studies that have uncovered important molecular mechanisms governing host-microbiota interaction.

\section{The study of host physiology using gnotobiotic fly model}

\section{A gnotobiotic fly model with classic genetics approach}

That the germ-free flies develop and grow at a slower pace is an old observation that has held true since Wollman's time. For example, in Baluka's monoxenic culture, the native bacterial isolates from the Drosophila gut, Stock 13, a Brevibacterium variant, accelerated pupariation compared to the axenic stock. ${ }^{[35]}$ This observation has now been further characterized in greater detail. On a "standard" laboratory diet, the pupariation and adult eclosion rate of the axenic flies are delayed by one day compared to their CR siblings. ${ }^{[23,42]}$ However, this delay becomes striking when the axenic flies are presented with nutritive challenges. Particularly, when raised on a diet where the yeast content was below $0.1 \%$, or was completely replaced by casamino acids, the germ-free flies died. ${ }^{[23]}$ This observation suggests that an intact gut microbiota provides life-sustaining factors for the host experiencing severe nutritive duress. Next, when fed on a diet with low yeast content, germ-free flies pupariate six days later than the CR flies. ${ }^{[24]}$ Therefore, the gut microbiota can also override the developmental delay to potentiate growth in suboptimal nutritive environment. Importantly, these two studies also demonstrated that inoculating the axenic fly embryos with one or several defined gut commensal species, such as Lactobacillus plantarum (L. plantarum) or Acetobacter pomorum (A. pomorum), can recapitulate the growth benefits conferred by the entire gut microbiota. Moreover, only certain strains of $L$. plantarum sustain growth on a 
low-yeast diet; several other isolates from the fly origin were unable to promote host growth even though they could colonize the larval gut and the fly food just as efficiently as the beneficial strains. ${ }^{[24]}$ This observation unequivocally illustrates that the gut microbiota promotes growth by not just serving as a food source, but through complex molecular and biochemical interactions with the host.

How then, does the gut microbiota promote host growth? First of all, like for many metazoan species, the source of the fly gut bacteria comes from contaminated food ${ }^{[19,29]}$ and naturally, some of the primary functions of the gut bacteria are to enhance digestion and expand the host's metabolic potential. The additional enzymatic activities of bacterial origin help break down the specific nutritive substrates that are otherwise indigestible for the host, who can in turn harvest energy from these food substrates and extract necessary metabolic building blocks for various biological processes. ${ }^{[6]}$ In addition, essential micronutrients derived from bacterial metabolism, such as vitamins and short-chain fatty acids, directly fuel the host's metabolism. ${ }^{[43]}$ Indeed, two recent studies found that fortifying the food fed to the germ-free flies with B vitamins phenocopies the effect of the presence of the gut bacteria to a large extent, indicating that the gut microbiota accomplishes metabolic sparing of the B vitamins for the host through a yet unknown mechanism. ${ }^{[42,44]}$

However, the growth benefits from the gut microbiota are probably beyond vitamin B provision. To identify the microbial factors that can rescue host lethality on the casein diet, Shin et al. conducted a random mutagenesis in A. pomorum and isolated strains that restored ex-germ-free larval survival on casamino acid diet but led to delayed pupariation when compared to animals mono-associated with the wild-type A. pomorum. Several such mutations affect pyrroloquinoline quinone-dependant alcohol dehydrogenase (Pqq-adh), an enzyme involved in the ethanol respiratory chain and whose end product is acetic acid. Although Pqq-adh mutant bacterial strains were impaired in their production of acetic acid, supplementation of casamino acid diet with acetic acid alone failed to rescue germ-free larval lethality. However, concomitant association with Pqq-adh mutant A. pomorum strains and supplementation with acetic acid completely rescued larval developmental timing. Therefore, upon severe nutritive challenge, the addition of $A$. pomorum first and foremost restores the viability of the fly host, and then the intact activity of the bacterial ethanol respiratory chain promotes host growth and maturation. Based on this result, it is likely that the molecular mechanisms that sustain larval life and promote growth are separable.

What are the host factors responding to the beneficial growth promotion effect of the microbiota in the presence of nutritional challenges? The studies of Shin et al. and
Storelli et al. demonstrate that the addition of A. pomorum or $L$. plantarum can accelerate growth and maturation by modulating host systemic hormonal signaling. In the Shin et al., study, larvae mono-associated with the Pqq-adh mutant strain of $A$. pomorum survived to adulthood, but displayed metabolic features reminiscent of defective insulin/ insulin-like growth factor (IIS) signaling, such as low body weight, retarded growth, elevated hemolymph glucose and trehalose levels, and higher level of triacylglyeride (TAG), the main form of stored lipids. At the molecular level, in the fat body of the flies mono-associated with mutant $P q q$-adh A. pomorum strains on the casamino diet, membrane activation of phosphoinositide 3-kinase (PI3K) and cytoplasmic retention of Drosophila forkhead box, subgroup O (dFOXO) were abolished, and the expression of insulin-like peptides (Dilps) such as Dilp3 and 5 was reduced in the larval brain. Most importantly, the ectopic expression of Dilp2 largely rescued both the defective IIS phenotype and the molecular signatures associated with such defects in flies mono-associated with mutant strain of $A$. pomorum. Therefore, A. pomorum, partly via its $p p q$-adh activity, regulates IIS to maintain the host's metabolic homeostasis [Figure 2]. Similarly, on a low-yeast diet, mono-association with L. plantarum lowered the expression of insulin receptor, a negative readout of pathway activity, suggesting that the presence of $L$. plantarum also enhances insulin signaling. ${ }^{[4]}$ Moreover, L. plantarum reduced the juvenile growth period through target of rapamycin (TOR) signaling: Dampening TOR activity in the fat body - the functional analogue of the mammalian liver - and the prothoracic gland compromised the L. plantarum growth-promoting effect as measured by adult emergence [Figure 2]. TOR is the host nutrient-sensitive signaling pathway devoted to balance organismal growth and maturation in a nutrient-dependent manner. ${ }^{[45,46]}$ In the developing larvae, TOR activity in the prothoracic gland directly controls ecdysone production, which in turn affects the parameters of systemic growth via IIS. As TOR responds to the circulating levels of different micronutrients in the hemolymph, such as branched-chain amino acids, L. plantarum may act upstream of TOR in several ways. First, $L$. plantarum can directly regulate TOR activity by making certain metabolites or other biochemical pathway intermediates and/or end products. Secondly, L. plantarum can either modify the diet or boost the host's digestive capacity to enhance nutrient assimilation, which then indirectly activates TOR pathway. Therefore, how L. plantarum promotes host juvenile growth is yet to be studied in detail.

Now two groups have demonstrated that specific strains from both Acetobacter and Lactobacillus families can promote juvenile growth upon nutritive challenge. What effect does the combined action of Acetobacter and Lactobacillus 


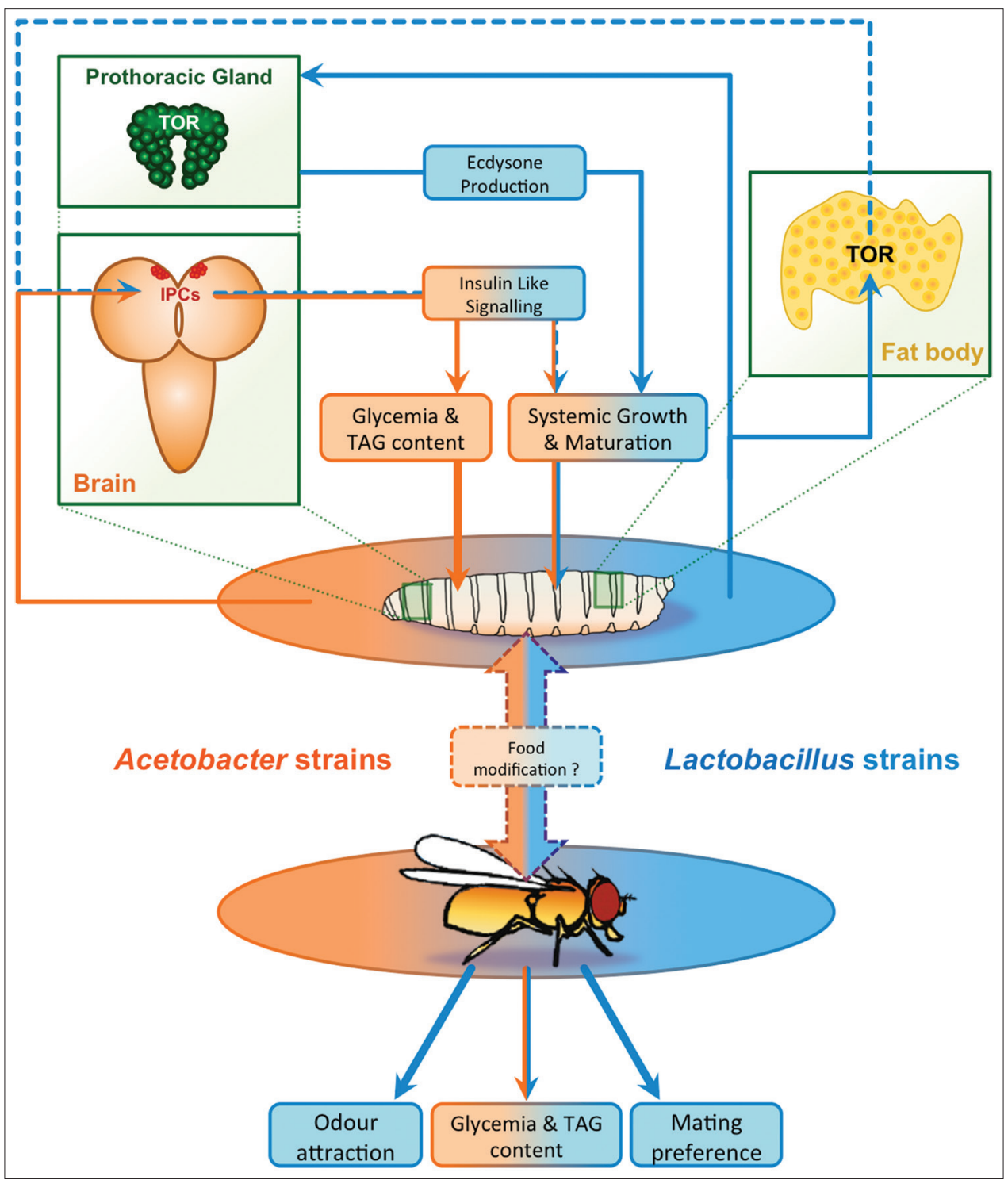

Figure 2: The host physiological and behavioral responses to the addition of different gut commensal strains. Gnotobiotic studies have depicted the effect of Acetobacter and Lactobacillus strains on systemic growth, metabolic homeostasis, and adult behavior. Specifically, in the presence of nutritive challenge, A. pomorum (orange) regulates host insulin signaling in the insulin-producing cells (IPCs) and thus promotes larval growth and maturation, whereas L. plantarum (blue) interacts with host target of rapamycin (TOR) pathway in the fat body and the prothoracic gland to control ecdysone production and affects insulin signaling directly or indirectly (dotted blue line). During the adult stage, both Acetobacter and Lactobacillus strains regulate host triacylglyceride (TAG) and circulating glucose levels, but only the Lactobacillus strains have been shown to impact host behaviors such as mating preference and odor attraction to food. The effect of the gut commensals represented by Acetobacter and Lactobacillus can be direct or through modifying the nutritional substrates.

have on the host? To study how these two commensal bacteria interact in the host and how such interactions impact adult host physiology, a study by Newell and Douglas compared differences in circulating glucose levels, TAG contents, and adult body weight between axenic flies and ex-germ-free flies associated with a single or different combinations of the five fly commensal species. ${ }^{[47]}$ Specifically, using a set of defined microbiomes with up to five commensal species (A. pomorum, Acetobacter tropicalis, L. plantarum, Lactobacillus brevis, and Lactobacillus fructivorans), the authors inoculated the germ-free flies with one or different combinations of these strains and found that all these combinations lowered the circulating glucose concentrations in comparison to axenic flies. However, in terms of lowering host TAG levels, these different combinations of bacteria worked with different efficiency. The Lactobacillus species could lower the TAG level moderately; Acetobacter did so more effectively than Lactobacillus, but was not as effective as the five species co-inoculation, which was the only treatment that recapitulated the benefits of the conventional 
commensal flora. Interestingly, one specific co-inoculation, with $A$. tropicalis and $L$. brevis, was particularly potent in that it lowered host TAGs more than in animals poly-associated with the five commensal species. Other forms of co-associations with two bacterial representatives from the Lactobacillus and Acetobacter genera failed to reproduce the phenotype. These results indicate that Acetobacter and Lactobacillus strains can act in synergy, but not consistently. A plausible explanation for this puzzling phenomenon is that the content of the microbiome, rather than the taxonomic combination, determines the TAG content of the host (see below).

\section{Gnotobiotic model with systems approach}

1.Large-scale identification of bacterial genetic determinants for mutualism

Using classic genetic analysis on a mono-association fly model, Shin et al. and Storelli et al. identified the entry points to further dissect the molecular dialogue between the host and the gut microbiota that alters host physiological traits. Gnotobiotic models are now deployed for large-scale search to fit the same purpose. In a metagenomic study, Chaston et al., first gathered a collection of 41 fully sequenced bacterial strains broadly encompassing different Acetobacter and Lactobacillus genus. Raised in mono-association with each of the 41 strains, the fly hosts showed a spectrum of different responses in terms of pupariation timing and adult TAG content. Based on the comparison of the amplitude of the mono-association effect on these two parameters, the authors undertook a metagenome-wide association study (MGWAS) that effectively correlates bacterial genetic determinants with the magnitude of changes in developmental timing and TAG content. ${ }^{[25]}$ Remarkably, the MGWAS based on developmental timing first yielded clusters of genes operating in the cellular respiratory chain, including the PQQ enzyme that converts sugar and alcohol substrates to acetic acid. This result corroborates the finding from the transposon screen in A. pomorum by Shin et al. Interestingly, Shin et al., recovered the Pqq mutant bacteria on a casein-only fly medium that causes lethality in germ-free flies, whereas the MGWAS was conducted on standard laboratory fly food, where the developmental delay in germ-free flies was subtle. At a glance, it is a bit surprising that both studies uncovered the same bacterial factor based on host developmental timing, a trait that varies drastically in the two experimental setups. It provocatively suggests there is a robust and canonical host interaction with bacterial ethanol respiratory chain products that cannot be masked by different host nutritional backgrounds. Such response has been shown to involve host insulin signaling. How such robust interaction is maintained in different nutritional backgrounds is an extremely interesting topic to explore. Moreover, in the Chaston et al. study, the clusters of genes that correlated with lower host TAG content are known to regulate redox sensing and glucose oxidation, such as glucose dehydrogenase $(\mathrm{GDH})$, gluconate-2 dehydrogenase $(\mathrm{GnDH})$, and a single domain oxidoreductase (SDR). Importantly, introducing these candidate genes into selected Acetobacter strains that lack these enzymes conferred the ability to the bacteria to reduce host TAG level. Furthermore, the authors observed that ectopic expression of GDH and $\mathrm{GnDH}$ concomitantly lowered glucose content in the media where the gnotobiotic flies were raised. These results strongly suggest that the gut microbiota can modulate host lipid storage and nutritional homeostasis through altering the nutrient composition of the food. Another intriguing observation from the study is that the clustering of bacterial strains based on the effect on host developmental timing and TAG level is largely unrestricted to the taxonomic structure of the bacteria. Hence, the collective genetic composition of the gut microbiome once again proves to be a more faithful predictor of host response than taxonomic classification. Now looking back, the finding by Chaston et al. probably also partially explains why Newell and Douglas observed inconsistent TAG lowering effect in flies associated with different combinations of Acetobacter and Lactobacillus strains (see the previous section). Altogether, this particular study raises a few interesting issues. For example, Chaston et al. propose that by modifying the food, bacterial glucose metabolism impacts the adult host's capacity to store lipid. Does this observation hold true in the developing larvae? The published studies seem to favor the likelihood, as Shin et al. unequivocally demonstrated that gnotobiotic larvae harboring mutant Pqq mutant Acetobacter strain show higher circulating sugar and triglyceride as a result of the compromise in the host insulin signaling activity [Figure 2]. If this is the case, do the bacteria directly elicit the host insulin response, or is such insulin response an indirect result of bacteria altering the glucose content of the food? These two possibilities are not mutually exclusive, but require further detailed mechanistic studies that either tease them apart or meld them together. So far, Chaston et al. have been unable to rescue developmental delay by ectopically expressing the enzymes involved in glucose oxidation, but such negative outcome is likely to imply that the interaction between host maturation and microbiota metabolism is more complex than we think.

\section{Transcriptomic studies of host response to gnotobiotic association}

The association with certain commensal species modulates host IIS and TOR signaling. What other kind of molecular changes take place in the host in the presence of the gut microbiota? To answer this question, several groups recently undertook microarray studies to compare the transcriptomic differences between the germ-free flies and their 
CR siblings at different age, and demonstrated that in the fly gut, the presence of the microbiota significantly alters the expression of a core set of genes that control transcription, gut structure, immunity, metabolism, signaling, and stress response $^{[28,48,49]}$ (reviewed in Erkosar and Leulier, 2014). ${ }^{[29]}$ Among these studies, Guo et al. extended the microarray finding and elegantly showed that in the aging fly gut, the transcription factor Foxo represses peptidoglycan recognition protein SC2 (PGRP-SC2), which subsequently leads to hyperactivation of Rel/NF- $\mathrm{KB}$ activity that is responsible for an intestinal dysbiosis phenotype. In addition to these studies, another noteworthy microarray analysis using poly-associated gnotobiotic flies identified a short but focused list of genes whose functions are enriched in digestion and primary metabolism. ${ }^{[48]}$ Erkosar et al. conducted the microarray study on ex-germ-free adult flies exposed to a defined set of commensal bacterial strains (A. pomorum, Commensalibacter intestini, L. brevis, and L. plantarum). First, the poly-association yielded certain genes that overlap with those found in the concomitant study by Broderick et al. using CR flies. ${ }^{[28]}$ Specifically, such poly-association markedly up-regulates the expression of a set of digestive enzymes and other genes involved in primary metabolism. This result reflects the conventional notion that gut bacteria assist in host digestive functions to effectively extract nutrients and energy from food. Intriguingly still, half of the poly-association up-regulated genes identified by Erkosar et al. were also involved in response to intestinal infection, and the majority of these genes are directly or indirectly under the control of Relish, the Drosophila orthologue of the mammalian NFKB factor, p105. ${ }^{[50]}$ This result once again corroborates the study by Broderick et al., who also observed that more than half of the up-regulated genes in the CR fly gut changed the expression pattern in Relish mutant flies. As Relish is essential to the interplay between host innate immunity and nutritional response, Erkosar et al. postulated the following scenario: The presence of commensal strains usually promotes the expression of a certain set of digestive enzymes and metabolic genes, but in the presence of an acute infection, a change in the host transcriptome is triggered, so that these microbiota-mediated metabolic genes are down-regulated to prepare for immune defense, and such change is mediated by Relish. Consistent with this hypothesis, the authors found that the expression patterns of several selected candidate genes such as trypsin and Jonah proteases are indeed down-regulated upon pathogen infection or in the genetic background where Relish activity is compromised. In summary, the finding by Erkosar et al. first largely recapitulates the host's transcriptomic response to gut microbiota in CR flies, thus cementing the utility and relevance of the poly-association model. Furthermore, like Broderick et al., the authors identified Relish as the central regulator of a transcriptional trade-off between metabolic response and immunity, and thus opened a new chapter for potential mechanistic studies of such switch. Altogether, the studies by Erkosar et al. and others were the first to demonstrate that the gut microbiota profoundly alters the host transcriptomic landscape, yet we know little how the bacteria mechanistically effect these changes. Secondly, these studies also provide an exhaustive list of genes that govern the host response to the gut microbiota. The functional studies of these candidates will immensely advance our understanding of the molecular basis of host-microbiota interaction. Furthermore, how do these host transcriptomic changes integrate into the known insulin and TOR signaling networks - as a response to the gut microbiota - to control systemic growth and metabolic homeostasis? Similarly, are these transcriptomic changes directly mediated by unknown bacterial factors, or through bacterial modification of the food substrate, or both? If both, what are the bacterial factors and how is the food modified? These are immediate questions that can be addressed with gnotobiotic models coupled to metabolomics and mutagenesis studies.

\section{The gut microbiota impacts social behavior}

Throughout the long eukaryotic evolutionary history, many animal species abandoned the solitary lifestyle for group living in highly developed social structures, in exchange for bodily protection, cooperative foraging, and increased chances of mating and reproduction. As the long-time evolutionary partner of its eukaryotic host, it is not surprising that the symbiotic gut bacteria also evolved to control host individual and social behavior, probably with the interest to maximize its transmission among the members of the society. ${ }^{[5]]}$ Through bidirectional signaling along the "microbiota-gut-vagus-brain axis", the activities of the gut microbiota can impact the activities of host neural circuitry and alter host foraging behavior, stress and anxiety response, and even the development of empathy. ${ }^{[51-53]}$ Gnotobiotic flies have recently emerged to be a productive model to study social interactions. For example, fruit flies preferentially mate with partners fed on the same kind of diet, a phenomenon termed "positive assortive mating", which is readily lost in axenic flies. However, the gnotobiotic addition of L. plantarum restores such positive assortive mating, indicating that the gut microbiota may play a direct role in altering fly pheromone composition according to the host's dietary environment. ${ }^{[54]}$ Besides mating preference, the presence of gut microbiota was also shown to determine how fruit flies are attracted by odors from different food substrates. ${ }^{[5]}$ In controlled learning experiments, Venu et al. presented the larvae subjects with three separate food choices: Fresh laboratory food, food processed by axenic larvae, and food used by CR larvae. While the larvae and adult female subjects 
showed no preference between the fresh food and axenically processed food, they were strongly attracted to the food substrate where the CR larvae were raised. Furthermore, the same larvae subjects equally preferred food that has been used to raise larvae mono-associated with $L$. brevis or L. plantarum. As an important control, the same larvae subject showed no preference to fly food containing only cultured $L$. brevis, indicating that the interaction between $L$. brevis or $L$. plantarum with the fly larvae is imperative to generate the source for such social attraction. The nature of such source is unknown, but it can be a volatile compound produced by either the bacteria or the larvae when both are residing in the same niche [Figure 2]. In the wild, fruit flies search of hospitable habitat with suitable food substrate for mating, egg laying, and rearing larvae. ${ }^{[56]}$ The results from this study imply that the host interaction with the commensal Lactobacillus genus of the gut microbiota can manufacture compounds that serve as cues for the host's searching effort and decision-making. What are these compounds? Through what pathways and neurons do they act? What other aspects of fly behavior do they affect? These are the questions that probably can also be answered with gnotobiotic studies.

\section{Conclusion}

In a recent essay, McFall-Ngai et al. commented that we humans are just "animals in a bacterial world". ${ }^{[3]}$ This pithy statement rightly illustrates the overwhelming number and the diversity of the microbes that we live with, yet we have only begun to grasp how these seemingly humble dwellers can powerfully change our being throughout evolution. By enhancing the host's metabolic potential, the gut microbiota helps expand the host's ecological niche. By altering host behavior, these bacteria probably also played their parts in shaping social hierarchies and caste systems in the animal kingdom. We still know very little about how the bacteria do it. However, by harnessing the power of the gnotobiotic flies, we have begun to systemically characterize how the gut microbiota potently elicits a myriad of host physiological responses and behavioral changes. Importantly, the gnotobiotic model, in combination with classic genetics and large-scale next-generation sequencing methods, grants us the unprecedented power of resolution to pinpoint the specific bacterial factors responsible a particular host phenotype. Only with such resolution, we can delve deeper into the mechanisms that govern host-microbiota interaction, and find answers to how these mechanisms evolved over time in different species. However, no matter how complex and unexpected these answers are, they never will deviate from the truth that Pasteur and Wollman prompted us to discover that our genetic makeup is metagenomic and our life story is indispensably, microbial.

\section{Acknowledgements}

The work in F.L. lab is funded by an ERC starting grant (FP7/2007-2013-N³09704), the ATIP/AVENIR program, the FINOVI fondation, the "Fondation Schlumberger pour l'Education et la Recherche". GS is sponsored by a fellowship from the "Fondation pour le recherche Medicale".

\section{REFERENCES}

1. Pasteur L. Observations related to the previous notes from M. Duclaux C.R. Acad Sci 1885;100:68.

2. Wollman E. On raising sterile flies. Contribution to the knowledge of the role of microbes in the digestive tract. Ann Inst Pasteur (Paris) $1911 ; 25: 79-88$.

3. McFall-Ngai M, Hadfield MG, Bosch TC, Carey HV, Domazet-Lošo T, Douglas AE, et al. Animals in a bacterial world, a new imperative for the life sciences. Proc Natl Acad Sci USA 2013;110:3229-36.

4. Douglas AE. Symbiosis as a general principle in eukaryotic evolution. J Mol Bio 20014;426:3830-7.

5. Human Microbiome Project Consortium. Structure, function and diversity of the healthy human microbiome. Nature 2012;486:207-14.

6. Sommer F, Bäckhed F. The gut microbiota - Masters of host development and physiology. Nat Rev Microbiol 2013;11:227-38.

7. Eckburg PB, Bik EM, Bernstein CN, Purdom E, Dethlefsen L, Sargent M, et al. Diversity of the human intestinal microbial flora. Science 2005;308:1635-8.

8. Clemente JC, Ursell LK, Parfrey LW, Knight R. The impact of the gut microbiota on human health: An integrative view. Cell 2012;148:1258-70

9. Sharon G, Garg N, Debelius J, Knight R, Dorrestein PC, Mazmanian SK. Specialized metabolites from the microbiome in health and disease. Cell Metab 2014;20:719-30.

10. Kaiko GE, Stappenbeck TS. Host-microbe interactions shaping the gastrointestinal environment. Trends Immunol 2014;35:538-48.

11. Grenham S, Clarke G, Cryan JF, Dinan TG. Brain-gut-microbe communication in health and disease. Front Physiol 2011;2:94.

12. Gibson MK, Pesesky MW, Dantas G. The yin and yang of bacterial resilience in the human gut microbiota. J Mol Biol 2014;426:3866-76.

13. O'Hara AM, Shanahan F. The gut flora as a forgotten organ. EMBO Rep 2006;7:688-93.

14. Karlsson F, Tremaroli V, Nielsen J, Bäckhed F. Assessing the human gut microbiota in metabolic diseases. Diabetes 2013;62:3341-9.

15. Tremaroli V, Bäckhed F. Functional interactions between the gut microbiota and host metabolism. Nature 2012;489:242-9.

16. Irrazábal T, Belcheva A, Girardin SE, Martin A, Philpott DJ. The multifaceted role of the intestinal microbiota in colon cancer. Mol Cell 2014;54:309-20.

17. Fond G, Boukouaci W, Chevalier G, Regnault A, Eberl G, Hamdani N, et al. The "psychomicrobiotic": Targeting microbiota in major psychiatric disorders: A systematic review. Pathol Biol (Paris) 2015;63:35-42.

18. Lemaitre B, Miguel-Aliaga I. The digestive tract of Drosophila 
melanogaster. Annu Rev Genet 2013;47:377-404.

19. Broderick NA, Lemaitre B. Gut-associated microbes of Drosophila melanogaster. Gut Microbes 2012;3:307-21.

20. Erkosar B, Storelli G, Defaye A, Leulier F. Host-intestinal microbiota mutualism: "Learning on the fly". Cell Host Microbe 2013;13:8-14.

21. Chandler JA, Lang JM, Bhatnagar S, Eisen JA, Kopp A. Bacterial communities of diverse Drosophila species: Ecological context of a host-microbe model system. PLoS Genet 2011;7:e1002272.

22. Ridley EV, Wong AC, Westmiller S, Douglas AE. Impact of the resident microbiota on the nutritional phenotype of Drosophila melanogaster. PLoS One 2012;7:e36765.

23. Shin SC, Kim SH, You H, Kim B, Kim AC, Lee KA, et al. Drosophila microbiome modulates host developmental and metabolic homeostasis via insulin signaling. Science 2011;334:670-4.

24. Storelli G, Defaye A, Erkosar B, Hols P, Royet J, Leulier F. Lactobacillus plantarum promotes Drosophila systemic growth by modulating hormonal signals through TOR-dependent nutrient sensing. Cell Metab 2011;14:403-14.

25. Chaston JM, Newell PD, Douglas AE. Metagenome-wide association of microbial determinants of host phenotype in Drosophila melanogaster. MBio 2014;5:e01631-14.

26. Wong CN, Ng P, Douglas AE. Low-diversity bacterial community in the gut of the fruitfly Drosophila melanogaster. Environ Microbiol 2011;13:1889-900.

27. Blum JE, Fischer CN, Miles J, Handelsman J. Frequent replenishment sustains the beneficial microbiome of Drosophila melanogaster. MBio 2013;4:e00860-13.

28. Broderick NA, Buchon N, Lemaitre B. Microbiota-induced changes in Drosophila melanogaster host gene expression and gut morphology. MBio 2014;5:e01117-14.

29. Erkosar B, Leulier F. Transient adult microbiota, gut homeostasis and longevity: Novel insights from the Drosophila model. FEBS Lett 2014;588:4250-7.

30. Chouaia B, Gaiarsa S, Crotti E, Comandatore F, Degli Esposti M, Ricci I, et al. Acetic acid bacteria genomes reveal functional traits for adaptation to life in insect guts. Genome Biol Evol 2014;6:912-20.

31. Crotti E, Damiani C, Pajoro M, Gonella E, Rizzi A, Ricci I, et al. Asaia, a versatile acetic acid bacterial symbiont, capable of cross-colonizing insects of phylogenetically distant genera and orders. Environ Microbiol 2009;11:3252-64.

32. Rastall RA. Bacteria in the gut: Friends and foes and how to alter the balance. J Nutr 2004;134:2022S-6S.

33. Reuter G. The Lactobacillus and Bifidobacterium microflora of the human intestine: Composition and succession. Curr Issues Intest Microbiol 2001;2:43-53.

34. Walter J, Britton RA, Roos S. Host-microbial symbiosis in the vertebrate gastrointestinal tract and the Lactobacillus reuteri paradigm. Proc Natl Acad Sci USA 2011;108 Suppl 1:4645-52.

35. Bakula M. The persistence of a microbial flora during postembryogenesis of Drosophila melanogaster. J Invertebr Pathol 1969;14:365-74.

36. Begg M, SANG JH. A method for collecting and sterilizing large numbers of Drosophila eggs. Science 1950;112:11-2.

37. Grönke S, Clarke DF, Broughton S, Andrews TD, Partridge L. Molecular evolution and functional characterization of Drosophila insulin-like peptides. PLoS Genet 2010;6:e1000857.
Using gnotobiotic flies to study host-microbiota interaction

38. Hamilton PT, Perlman SJ. Host defense via symbiosis in Drosophila. PLoS Pathog 2013;9:e1003808.

39. Ikeya T, Broughton S, Alic N, Grandison R, Partridge L. The endosymbiont Wolbachia increases insulin/IGF-like signalling in Drosophila. Proc Biol Sci 2009;276:3799-807.

40. Ringo J, Sharon G, Segal D. Bacteria-induced sexual isolation in Drosophila. Fly (Austin) 2011;5:310-5.

41. Ridley EV, Wong AC, Douglas AE. Microbe-dependent and nonspecific effects of procedures to eliminate the resident microbiota from Drosophila melanogaster. Appl Environ Microbiol 2013;79:3209-14.

42. Wong AC, Dobson AJ, Douglas AE. Gut microbiota dictates the metabolic response of Drosophila to diet. J Exp Biol 2014;217:1894-901.

43. Natarajan N, Pluznick JL. From microbe to man: The role of microbial short chain fatty acid metabolites in host cell biology. Am J Physiol Cell Physiol 2014;307:C979-85.

44. Fridmann-Sirkis Y, Stern S, Elgart M, Galili M, Zeisel A, Shental N, et al. Delayed development induced by toxicity to the host can be inherited by a bacterial-dependent, transgenerational effect. Front Genet 2014;5:27.

45. Danielsen ET, Moeller ME, Rewitz KF. Nutrient signaling and developmental timing of maturation. Curr Top Dev Biol 2013;105:37-67.

46. Hietakangas V, Cohen SM. Regulation of tissue growth through nutrient sensing. Annu Rev Genet 2009;43:389-410.

47. Newell PD, Douglas AE. Interspecies interactions determine the impact of the gut microbiota on nutrient allocation in Drosophila melanogaster. Appl Environ Microbiol 2014;80:788-96.

48. Erkosar B, Defaye A, Bozonnet N, Puthier D, Royet J, Leulier F. Drosophila microbiota modulates host metabolic gene expression via IMD/NF-кB signaling. PLoS One 2014;9:e94729.

49. Guo L, Karpac J, Tran SL, Jasper H. PGRP-SC2 promotes gut immune homeostasis to limit commensal dysbiosis and extend lifespan. Cell 2014;156:109-22.

50. Buchon N, Broderick NA, Poidevin M, Pradervand S, Lemaitre B. Drosophila intestinal response to bacterial infection: Activation of host defense and stem cell proliferation. Cell Host Microbe 2009;5:200-11.

51. Montiel-Castro AJ, González-Cervantes RM, Bravo-Ruiseco G, Pacheco-López G. The microbiota-gut-brain axis: Neurobehavioral correlates, health and sociality. Front Integr Neurosci 2013;7:70.

52. Cryan JF, Dinan TG. Mind-altering microorganisms: The impact of the gut microbiota on brain and behaviour. Nat Rev Neurosci 2012;13:701-12.

53. Stilling RM, Dinan TG, Cryan JF. Microbial genes, brain and behaviour-Epigenetic regulation of the gut-brain axis. Genes Brain Behav 2014;13:69-86.

54. Sharon G, Segal D, Ringo JM, Hefetz A, Zilber-Rosenberg I, Rosenberg E. Commensal bacteria play a role in mating preference of Drosophila melanogaster. Proc Natl Acad Sci USA 2010;107:20051-6.

55. Venu I, Durisko Z, Xu J, Dukas R. Social attraction mediated by fruit flies’ microbiome. J Exp Biol 2014;217:1346-52.

56. Durisko Z, Dukas R. Attraction to and learning from social cues in fruitfly larvae. Proc Biol Sci 2013;280:20131398. 